\title{
Automated Measurement and Monitoring of the Electromagnetic Fields from GSM Systems
}

\author{
Eduard Lunca, Member, APCBEES, Alexandru Salceanu, and Silviu Ursache
}

\begin{abstract}
The main objective of the present study is to introduce a virtual instrumentation system for automated characterization of the electromagnetic fields generated by GSM systems. The system consists of calibrated antennas, remotely controlled spectrum analyzer with USB connectivity and dedicated LabVIEW software for data transfer, processing, analysis and monitoring. Primarily intended to determine the RF fields from GSM base stations, it can also be used for investigating the RF exposure from other communication technologies.
\end{abstract}

Index Terms-Automated measurements, GSM systems, LabVIEW graphical programming, RF electromagnetic fields.

\section{INTRODUCTION}

During the past decade, the increased use of mobile phones has led to the installation of an extremely large number of mobile phone base stations, especially in densely populated areas. As a consequence, public concern about possible adverse health effects of the exposure to RF electromagnetic fields generated by GSM systems has often emerged. Therefore, there is a need not only for computing the RF fields from base station antennas [1]-[3], but also for performing systematic measurements of the RF exposure levels in their vicinity [3]-[6], to ensure that these levels are within the reference limits specified in applicable guidelines.

Because, in most cases, the assessment of RF exposure from GSM systems require carrying out and processing a large volume of measurements, our efforts have been directed towards the development of an automated solution for RF field strength characterization. By adopting a virtual instrumentation approach focused on LabVIEW graphical programming, it was possible to combine traditional equipment with "measurement" algorithms into a more flexible PC-based system, which can compute, analyze and monitor various parameters, such as electric field strength (E), magnetic field strength $(\mathrm{H})$, power density $(\mathrm{S})$, mean and standard deviation of these quantities, etc.

The main aspects regarding the system operation and software functionality, as well as a few short-term monitoring results, are presented in the subsequent sections.

Manuscript received December 20, 2012; revised January 22, 2013. This paper was supported by the project PERFORM-ERA "Postdoctoral Performance for Integration in the European Research Area” (ID-57649), financed by the European Social Fund and the Romanian Government.

The authors are with the Faculty of Electrical Engineering, Department of Electrical Measurements and Materials, Iasi, Romania (e-mail elunca@ee.tuiasi.ro; asalcean@ee.tuiasi.ro; silviu_ursache@ee.tuiasi.ro).

\section{VIRTUAL INSTRUMENTATION SYSTEM}

The virtual instrumentation system (VIS) is built on a PC program and an R\&S FS300 programmable spectrum analyzer, which operates in the frequency range from $9 \mathrm{kHz}$ to $3 \mathrm{GHz}$. With 16 digitally implemented resolution bandwidths from $200 \mathrm{~Hz}$ to $1 \mathrm{MHz}$, the R\&S FS300 can be optimally configured to perform narrowband measurements of the RF-fields generated by both GSM 900 and GSM 1800 base station antennas. Unfortunately, this instrument has no RMS detector (it has only positive peak detector), which is usually preferred when assessing the RF exposure originating on UMTS systems [7], [8].

Because the R\&S FS300 has low power requirements and provides full remote control via USB, the VIS can be used for performing both indoor and outdoor measurements. In the last case, a notebook computer and a common uninterruptible power supply (UPS) for home or office could provide a simple solution for ensuring portability. Such a setup is shown in Fig. 1, where the R\&S FS300 is operated in conjunction with an AT4002A, $800 \mathrm{MHz}-5 \mathrm{GHz}$ horn antenna (Amplifier Research) for measuring E-field emissions in the vicinity of a multi-system base station from an urban environment.

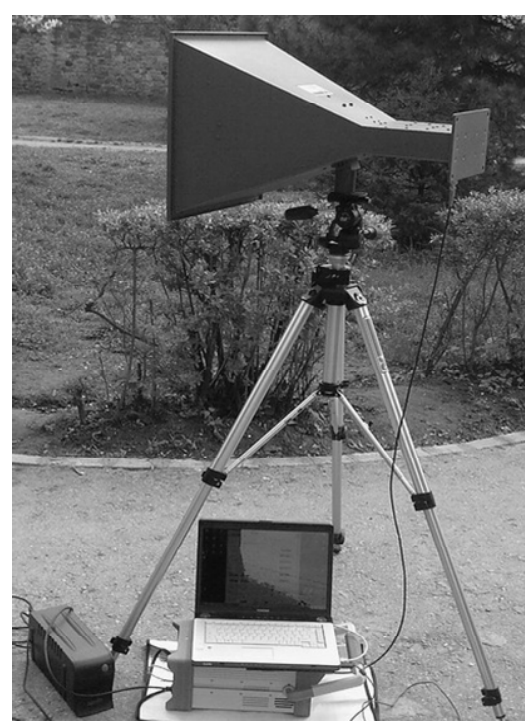

Fig. 1. Hardware configuration for outdoor measurements.

Note that, for indoor locations with Internet connectivity, the system can be configured to provide remote control through the LabVIEW built-in Web Server [9]. In this way, the front panel of the application can be viewed and controlled from a web browser, by using only the LabVIEW Run-Time Engine - a small program downloadable on the National Instruments' website. 


\section{SOFTWARE}

The associated software is designed to work under Windows XP and - as already stated - has been created as a LabVIEW virtual instrument (VI). Therefore, it takes advantage of numerous benefits provided by this environment, including interactive graphical user interfaces, advanced data visualization, intuitive operation and possibility of further expansion. When running the software, the operation of the spectrum analyzer through its front panel is disabled and only a "blue screen" will be displayed by the instrument.

Among others, the program - called RF-FieldVIEW - is responsible for configuring the main parameters of the spectrum analyzer, retrieving the power data from the instrument, computing and displaying the field strength, time-averaged exposure and a number of statistic parameters, as well as recording data for subsequent analysis. The stored data can be analyzed by using another LabVIEW program, which also allows for computing the resultant RMS field by taking into account readings recorded on three orthogonal directions for a sufficiently long measurement time, as suggested in [10].

\section{A. Algorithm Summary}

Basically, the RF-FieldVIEW software is based on the LabVIEW instrument driver library for the FS300, provided by Rohde \& Schwarz on its website. All these functions have been critically studied and a part of them have been incorporated as subVIs in the main program for ensuring proper control of the spectrum analyzer and receiving the trace data from the instrument, as presented in Fig. 2. Starting from the "live" trace data, further LabVIEW processing is devoted to compute relevant field quantities and statistic parameters.

In order to provide simple system reconfiguration, the RF-FieldVIEW uses a modular approach. For instance, a number of subroutines (subVIs) are specifically designed for adding the antenna factors to the measurement results and compensating for the transmission line losses over the frequency range explored by the spectrum analyzer. If a particular antenna or transmission line must be used, the correspondent subVI will be called by the main program when it starts and no code modifications have to be made.

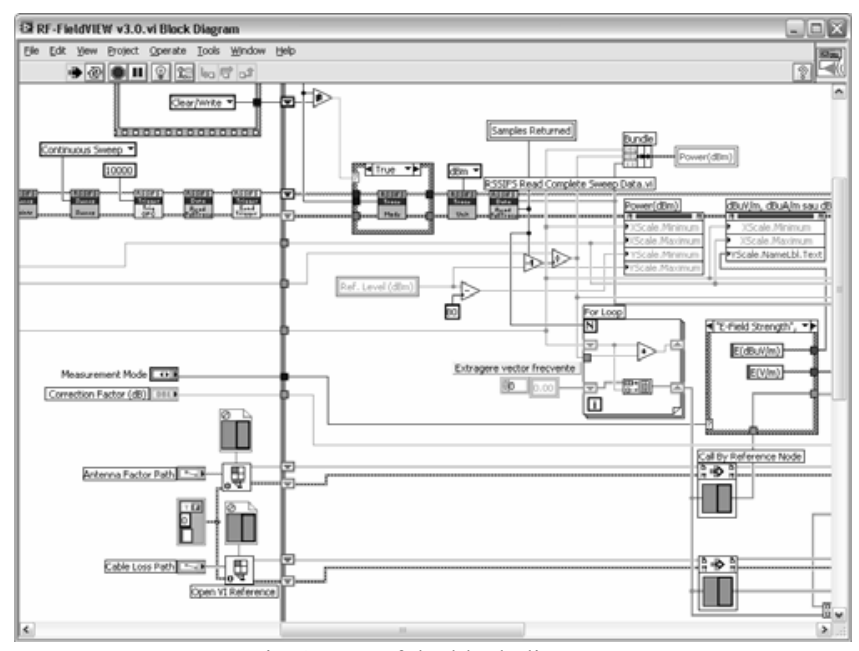

Fig. 2. Part of the block diagram.
The program runs continuously until the user aborts its execution, a specified execution time has elapsed or a communication error has been detected. In this case, an error message will be displayed for the user.

\section{B. Graphical User Interface (GUI)}

The graphical user interface of the RF-FieldVIEW software is organized on six tabbed windows, whose functionality will be presented in the following, and a "configuration" section that is initially used for selecting the measurement type (E, H or S), loading the calibration data of the measurement antenna and eventually specifying additional corrections (if they are known). This section of the GUI also contains options for activating and configuring the record function, either before or during the measurement process.

Fig. 3 shows the "Analyzer" window, which allows for setting the parameters of the spectrum analyzer (frequency span, reference level, resolution bandwidth, video bandwidth, etc.) and displaying the "live" power spectrum of the RF input signal, in units of $\mathrm{dBm}$. The graphical display for power simulates the screen of the FS 300 and automatically adapts to the user inputs. After the program starts, the evaluation mode of the power trace - clear/write, view, max hold, min hold and average - can be changed at any moment.

Depending on the measurement type, the program displays either the electric field strength, in $\mathrm{V} / \mathrm{m}$ and $\mathrm{dB} \mu \mathrm{V} / \mathrm{m}$, magnetic field strength, in $\mathrm{A} / \mathrm{m}$ and $\mathrm{dB} \mu \mathrm{A} / \mathrm{m}$, or power density, in $\mathrm{mW} / \mathrm{m}^{2}$ and $\mathrm{dBm} / \mathrm{m}^{2}$. Because $\mathrm{E}, \mathrm{H}$ and $\mathrm{S}$ are derived from power, changing the evaluation mode of the power trace also affects any of these readings. As it can be observed in Fig. 4, the E, $\mathrm{H}$ and $\mathrm{S}$ traces can be directly compared with relevant exposure limits and, as in the case of the power spectrum, analyzed with markers.

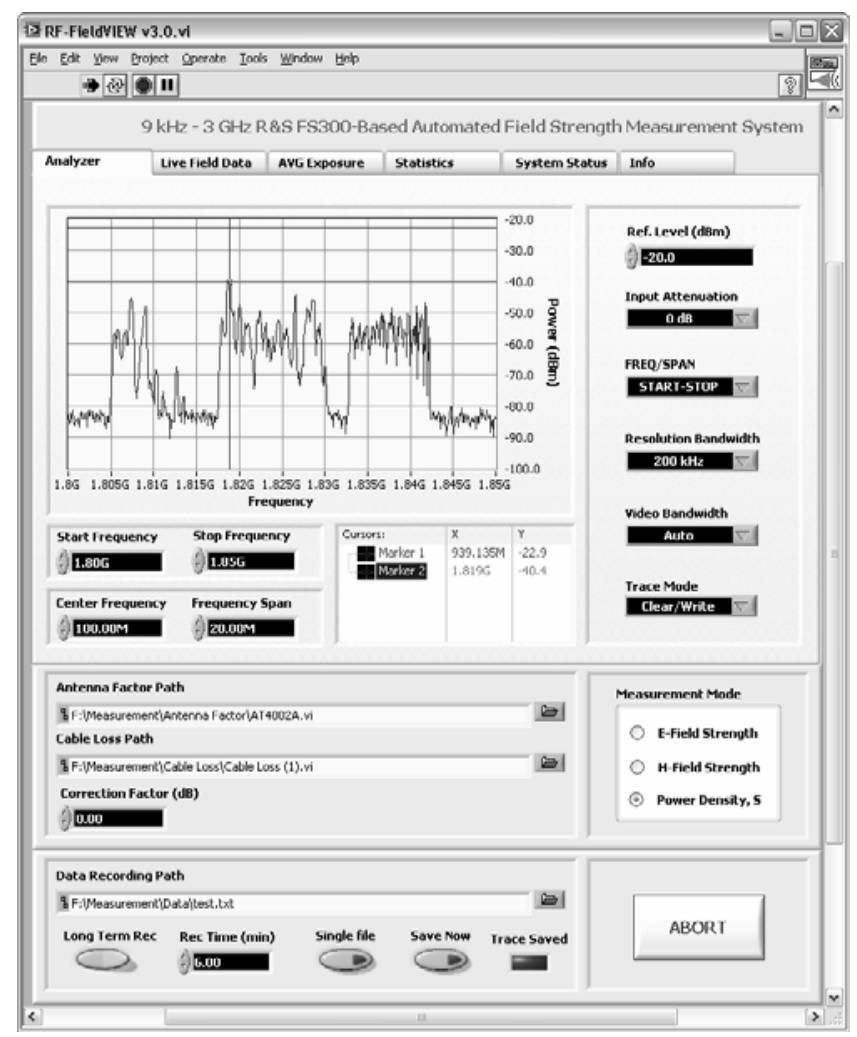

Fig. 3. The "Analyzer" window 


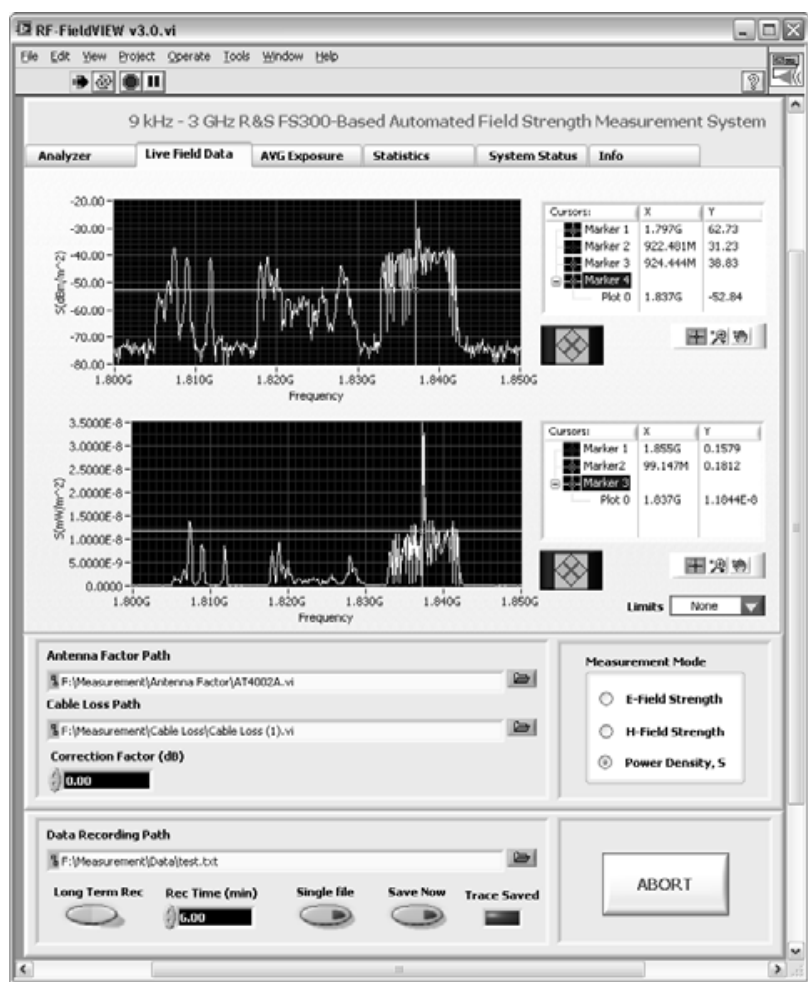

Fig. 4. The "Live Field Data" window.

If desired, the "AVG Exposure" window allows for applying time averaging to $\mathrm{E}, \mathrm{H}$ and $\mathrm{S}$, usually over 6 minutes or 30 minutes, but both the averaging time and sample time are programmable. Additionally, the "Statistics" window could be used to perform statistical calculations on the field data, generating four dynamic spectra representing the min, max, mean and standard deviation of the individual frequency bins over a desired frequency range. Finally, the "System Status" window provides settings for hardware initialization and reports potential errors, whereas the "Info" window provides useful information regarding system operation, emission types and related settings, amplitude corrections to be applied, etc.

For applications requiring field surveillance, the program can be configured to record virtually any volume of time-stamped field traces on the PC's hard disk. The recorded data can be analyzed with either a dedicated LabVIEW program, similar to that described in [11], or spreadsheet software, such as MS Excel.

\section{MONITORING RESUlts}

According to the ICNIRP guidelines [12], the reference levels for general public exposure from mobile communications are: $4.5 \mathrm{~W} / \mathrm{m}^{2}$ for GSM networks operating in the $900 \mathrm{MHz}$ frequency band, $9 \mathrm{~W} / \mathrm{m}^{2}$ for GSM networks operating at $1800 \mathrm{MHz}$ and $10 \mathrm{~W} / \mathrm{m}^{2}$ for $3 \mathrm{G} / \mathrm{UMTS}$. In order to get an idea of the actual RF exposure levels associated with these technologies, a short-term monitoring has been conducted in a typical indoor environment.

First of all, to investigate the short-term evolution of the background electromagnetic field, wideband measurements from $800 \mathrm{MHz}$ to $2.6 \mathrm{GHz}$ have been taken at each 0.5 minutes during 24 hours (Fig. 5). Then, narrowband measurements covering the downlink frequency bands of the existing mobile phone networks have also been recorded at regular time intervals.

Fig. 6 shows the power density in the frequency range from $925 \mathrm{MHz}$ to $960 \mathrm{MHz}$, which covers both the E-GSM 900 and P-GSM 900 downlinks. This plot consists of 477 instantaneous readings taken over 1.5 hours and exhibits a maximum of $0.0015 \mathrm{~mW} / \mathrm{m}^{2}$ at the frequency of $940 \mathrm{MHz}$.

Similarly, Fig. 7 shows the power density in the frequency range from $1800 \mathrm{MHz}$ to $1850 \mathrm{MHz}$, which is within the frequency band associated with the DCS 1800 downlink. This plot consists of 740 instantaneous readings taken over 2.5 hours and exhibits a maximum of $0.0003 \mathrm{~mW} / \mathrm{m}^{2}$ at the frequency of $1818.9 \mathrm{MHz}$.

The power density in the frequency range from $2110 \mathrm{MHz}$ to $2170 \mathrm{MHz}$, which corresponds to the UMTS downlink, is shown in Fig. 8. This plot consists of 2030 readings taken over 17 hours, in the average mode, and has a maximum of $0.0391 \mathrm{~mW} / \mathrm{m}^{2}$ at the frequency of $2161.4 \mathrm{MHz}$. All measurements have been automatically corrected by a factor that accounts for the limited resolution bandwidth of the spectrum analyzer with respect to the UMTS signal bandwidth.

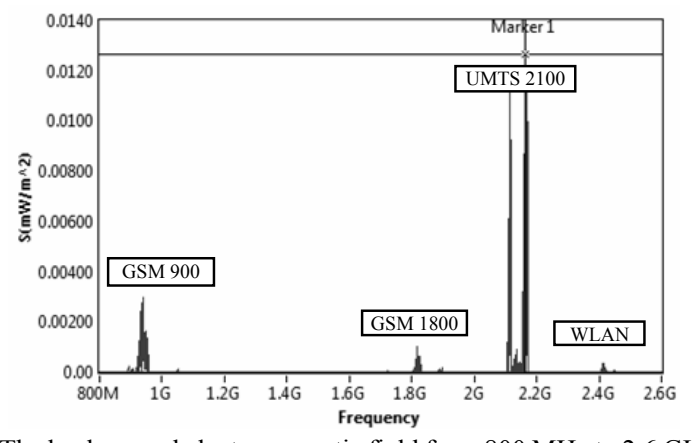

Fig. 5. The background electromagnetic field from $800 \mathrm{MHz}$ to $2.6 \mathrm{GHz}-24$ hours survey

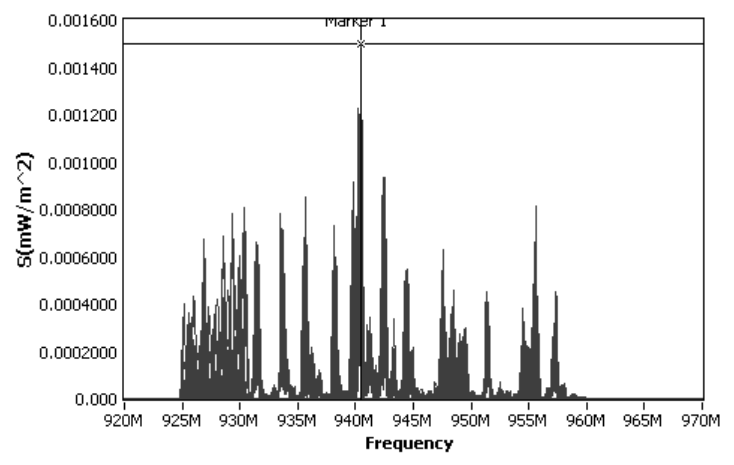

Fig. 6. Emissions from GSM 900.

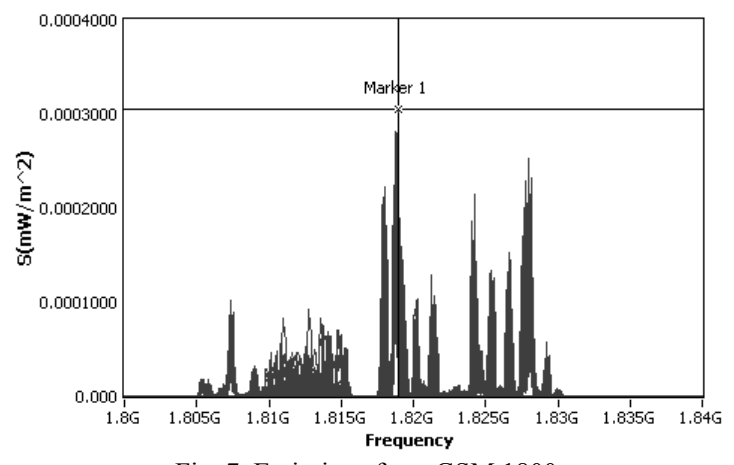

Fig. 7. Emissions from GSM 1800. 


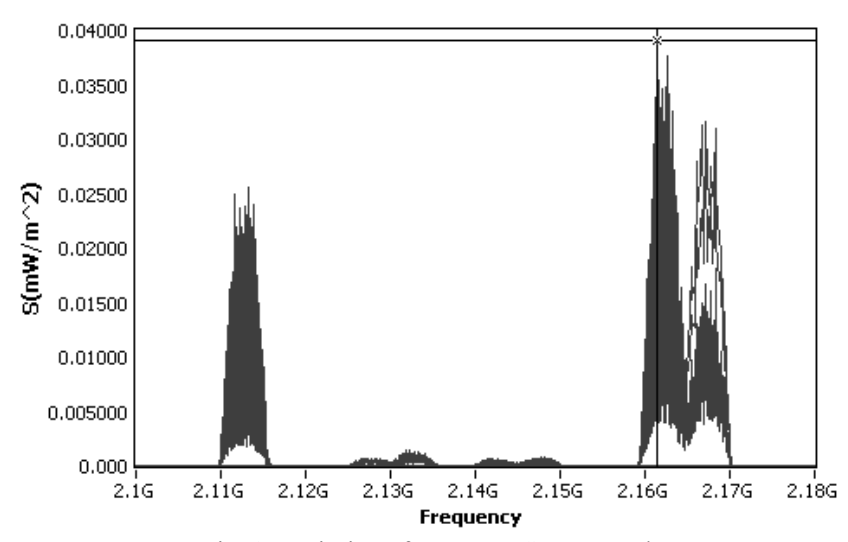

Fig. 8. Emissions from UMTS, averaged.

\section{CONCLUSION}

This work demonstrated that, using a LabVIEW graphical programming approach, it is possible to integrate a programmable spectrum analyzer into an automated system for measuring and monitoring the RF electromagnetic fields from GSM communication systems. The associated software (including that for off-line data analysis) provides user-friendly operation and accommodates various features for fast field characterization, including spectrum view for all quantities, markers, zoom, limits, simultaneous display of a very large number of saved traces, etc. Also, it can be easily updated with additional functionality and adapted to new requirements.

\section{REFERENCES}

[1] B. Kamo, R. Miho, V. Kolici, S. Cela, and A. Lala, "Estimation of peak power density in the vicinity of cellular base stations, FM, UHF and WiMAX antennas," International Journal of Engineering \& Technology, vol. 11, no. 2, pp. 65-71, 2011.

[2] D. Wojcik, "Evaluation of near field of the GSM base station antennas in urban environment," in Proc. 14th International Conference on Microwaves, Radar and Wireless Communications, Gdansk, 2002, pp. 191-194.

[3] S. Miclaus and P. Bechet, "Estimated and measured values of the radiofrequency radiation power density around cellular base stations," Romanian Journal of Physics, vol. 52, no. 3-4, pp. 429-440, 2007.

[4] P. Baltrènas and R. Buckus, "Indoor measurements of the power density close to mobile station antenna", in Proc. 8th International Conference "Environmental Engineering”, Vilnius, 2011, pp. 16-21.

[5] D.A. Shalangwa, "Measurement of exposure of radio frequency field (RF) radiation from global system for mobile communication (GSM) masts," Journal of Electrical and Electronics Engineering Research, vol. 2, no. 3, pp. 75-84, 2010.

[6] M. Ibrani, L. Ahma, E. Hamiti, and R. Sefaj, "Exposure assessment in the vicinity of $900 \mathrm{MHz}$ GSM base station antenna," in Proc. 11th WSEAS International Conference on Communications, Crete, 2007, pp. 139-143.

[7] G. Neubauer, K. Lamedschwandner, S. Cecil, and G. Schmid, "Exposure assessment methods for emerging new technologies," in Proc. XXIX URSI General Assembly, Chicago, 2008, pp. 1-4.

[8] C. Bornkessel and M. Wuschek. (2006). Exposure measurements of modern digital broadband radio services. in Proc. GeMiC 2006 German Microwave Conference. Karlsruhe. pp. 1-4.

[9] National Instruments. (December 5, 2007). Developing remote front panel labview applications. Tutorial. [Online]. Available: http://www.ni.com/white-paper/3277/en.

[10] W. Müllner, G. Neubauer, and H. Haider. (2002). Precise power flux density measurements at base stations. Compliance Engineering -
Annual Reference Guide. pp. 72-77. [Online]. Available: http://www.ce-mag.com/archive/02/Spring/mullner.html.

[11] E. Lunca and A. Salceanu, "Virtual instrumentation approach for teaching EMC concepts," Electronics and Electrical Engineering, vol. 117 , no. 1, pp. 75-80, 2012.

[12] ICNIRP. (1998). Guidelines for limiting exposure to time-varying electric, magnetic and electromagnetic fields (up to $300 \mathrm{GHz}$ ). Health Physics. vol. 71. no. 4. pp. 494-522. [Online]. Available: http://www.icnirp.de/documents/emfgdl.pdf.

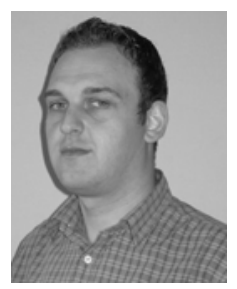

Eduard Lunca was born in Piatra-Neamt, Romania, on May 6, 1978. In 2001, he graduated from the "Gheorghe Asachi" Technical University of Iasi, Faculty of Electrical Engineering, study program - Metrology and Measurement Systems. In 2002, he obtained the Master's degree in Information Systems for Environmental Monitoring and started to work, as a teaching assistant, at the Faculty of Electrical Engineering, Department of Electrical Measurements and Materials, 21-23 Dimitrie Mangeron Street, 700050 , Iasi, Romania. PhD graduated in 2008, from the "Gheorghe Asachi" Technical University of Iasi, study program - Electrical Engineering.

Since 2009, he is a lecturer with the same department. His fields of interest include: EMC/ESD, EMF measurement and monitoring, electromagnetic modeling, analog and digital circuit design, and virtual instrumentation.

Dr. Lunca is a member of Romanian EMC Association and International Association of Online Engineering.

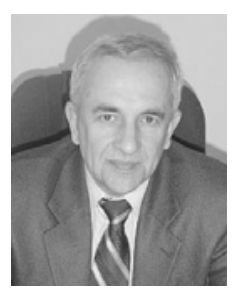

Alexandru Salceanu was born in Iasi, Romania, on Novemeber 14, 1955. In 1980, he graduated from the "Gheorghe Asachi" Technical University of Iasi, Faculty of Electrical Engineering. In 1997, the "Gheorghe Asachi" Technical University of Iasi conferred him the $\mathrm{PhD}$ degree in Electrical Measurements.

From 1980 to 1984 , he worked at the "Nicolina Factory" in Iasi, as a design engineer in the automation laboratory. Between 1984 and 1990, he was a research engineer with the Institute of Technical Physics, Iasi. In 1990, he started to work, as a teaching assistant, at the Faculty of Electrical Engineering, 21-23 Dimitrie Mangeron Street, 700050, Iasi, Romania. In October 2002, he was confirmed as a professor. Research fields: EMC/ESD, soft magnetic materials - measurements and phenomenological models, electrical measurements.

Prof. Salceanu is a member of Institute of Electrical and Electronic Engineers - EMC Society, International Measurement Confederation Technical Committee 4, Romanian Measurement Society, Romanian Standards Association - Technical Committee 30, etc.

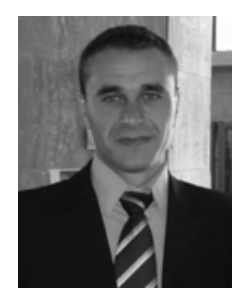

Silviu Ursache was born in Iasi, Romania, on January 24, 1983. In 2006, he graduated from the "Gheorghe Asachi" Technical University of Iasi, Faculty of Electrical Engineering, study program Metrology and Measurement Systems. In 2007, he obtained the Master's degree in Information Systems for Environmental Monitoring. PhD graduated in 2011, from the "Gheorghe Asachi" Technical University of Iasi, study program - Electrical Engineering.

He is currently working as a teaching assistant with the Faculty of Electrical Engineering, Department of Electrical Measurements and Materials, 21-23 Dimitrie Mangeron Street, 700050, Iasi, Romania. His fields of interest include: ESD, EMF measurement and monitoring, and applied mathematics in electrical engineering. 